\title{
Neuroprotective role of Glycyrrhiza glabra on chronic noise induced changes in Hippocampal morphology and its functions in wistar albino rats
}

\author{
Research Article
}

\begin{abstract}
Archana Arjunan1, Dhiraj Kumar Sah ${ }^{2}$, Ravindran Rajan ${ }^{3 *}$
1. Research Scholar, Department of Physiology, Dr. Arcot Lakshmanaswamy Mudhaliar Institute of Basic Medical Sciences, University of Madras, Taramani, Chennai.

2. Research Scholar, Research Institute of Medical Sciences, Chonnam National University Medical School, Gwangju 501-190, South Korea.

3. Associate Professor \& Head, Department of Physiology, Dr. Arcot lakshmanaswamy Mudhaliar Institute of Basic Medical Sciences, University of Madras, Taramani, Chennai.
\end{abstract}

\begin{abstract}
Background: Noise has become inexorable stress due to the increase in urbanization, automobile usage, and lifestyle modification.Aim: The study aims to investigate the effect of chronic noise stress on hippocampal morphology and the neuroprotective effect of Glycyrrhiza glabra (GG) on stress-induced male Wistar albino rats. Methods: Adult albino rats were randomly divided into four groups. Each group contained six animals. Rats exposed to chronic noise stress (100 dB/4 h - 30Days), Noise + GG (150 mg/kg Bw/Oral), and GG alone were compared with control animal and assessed for behaviour using the hole-board test, marble burying test, the morphology of hippocampus by histology, DNA fragmentation and assessed the Phytochemical constitutes in GG. Results: The rats exposed to chronic noise stress showed significance $(\mathrm{p}<0.05)$ of behavioral alterations such as increased fear and anxiety, obsessive-compulsive behaviour, enlarged lateral ventricle, and reduced hippocampal volume. Conclusion: The results reported that chronic noise stress affects the neurobehavioral due to reduced hippocampal volume.
\end{abstract}

Key Words: Anxiety, Fear, Glycyrrhiza glabra, Hippocampus, Lateral ventricle, Noise, Obsessive-compulsive, Stress.

\section{Introduction}

All living creatures depend on their environments for energy and materials that help in sustaining life. Although the environment sustains human life, it can also cause diseases(1). The health impacts caused by excessive environmental noise are a growing concern. Noise exposure has been linked to a variety of health effects, including hearing loss, cardiovascular damage, cognitive impairment, and sleep disturbance and annoyance(2). Enormous studies have shown that noise above $90 \mathrm{~dB}$ increases the stress hormones and can cause significant impact on human health. The brain is the target organ for corticosteroids(3) and the hippocampus is vulnerable to a number of insults and is prone to excitotoxic activity(4). Receptors for glucocorticoids are found in the hippocampus, amygdala and frontal cortex, these three brain regions are involved in-memory processing, and emotional regulation(3), (5).In the absence of effective neuro-protective drugs in modern

* Corresponding Author:

Ravindran Rajan

Associate Professor \& Head, Department of Physiology, Dr. Arcot lakshmanaswamy Mudhaliar Institute of Basic Medical Sciences, University of Madras. Taramani, Chennai - 113. India.

Email Id: ravindran89@gmail.com medicine, several medicinal plants in traditional medicine, like Glycyrrhiza glabraL. (GG), has been used to cure and prevent neuronal diseases. Plants are rich in a variety of compounds. In ayurvedic traditional system of medicine, Glycyrrhiza glabra (GG) (family: Leguminosae) is used as the anti-inflammatory, antiulcer, expectorant, antimicrobial and anxiolytic(6), (7)but lack of studies about neuroprotective effect of GG on stress-induced hippocampal dysfunctions. From this, it was planned to study the effect of chronic noise on hippocampal functions in a noise-induced animal model and to underpinning the role Glycyrrhiza glabra on stress induced hippocampal damage in wistar albino rats.

\section{Materials and Methods Animals}

Experimental animals were all healthy and weighed about $140-180 \mathrm{~g}$. The animals were reared in the Animal House of the Institute, University of Madras, Taramani, Chennai, India, and all the animals were maintained under standard laboratory conditions housed at 3 per cage $(29 \mathrm{~cm} \times 22 \mathrm{~cm} \times 14 \mathrm{~cm})$ and constant ambient temperature with $12-\mathrm{h}$ dark photoperiod; the rats were allowed free access to food and water. Appropriate ethical clearance was obtained for this work from the Institutional Animal Ethical Committee (IAEC no. 02/06/2019 dated 12/03/2019) 
prior to the experiments. Animals were divided into four groups, with six animals in each group.

- Group I - Control

- Group II - Chronic Noise stress (100dBA- 4 Hours / 30 Days)

- Group III - Chronic Noise Stress + GG (150 mg/Kg BW Oral)

- Group IV - GG alone (150 mg/Kg BW Oral)

\section{Noise Stress Induction}

When noise exposure exceeds 100dBA, noise becomes a stressor. Noise was produced by two loudspeakers $(15 \mathrm{~W})$, driven by a white-noise generator $(0-26 \mathrm{kHz})$, and installed $30-\mathrm{cm}$ above the cage. Animal were then exposed for $100 \mathrm{dBA} / 4$ hours per day for 30 days(8).

\section{Preparation and Administration of Ethanolic Root Extract of GG}

GG was purchased from National Institute of Siddha, Chennai. Dried roots wereground and about 50 $\mathrm{g}$ of dried root of GG powder was packed in thimble flask and $550 \mathrm{ml}$ ofethanol $(70 \%)$ was added in $1000 \mathrm{ml}$ round bottom flask. Then the soxhlet apparatus was set up to complete 10 to 15 cycles. After that the root extract was filtered and filtrate was concentrated up to $50 \mathrm{ml}$ using water bath at $60^{\circ} \mathrm{C}$. The concentrated extract was taken in evaporating dish (Borosilicate glass) which is previously weighed. Then the extract was evaporated till the thick liquor obtained after that it was dried. The yield of GG ethanolic extract was 12.15 $\%(\mathrm{w} / \mathrm{w})$ and stored in the freezer. Before the oral administration of extracted drug to animals it was dissolved in saline. Based on the animal weight $150 \mathrm{mg} /$ $\mathrm{kg}$ was administered orally.

\section{Quantitative analysis on phytochemical constituents}

The extracts obtained were subjected to qualitative chemical tests to detect the presence of various chemical constituents. Major secondary metabolites classes such as flavonoids(9), phenolics(10), and Carotenoid(11)was measured.

\section{Behavioral analysis \\ Marble Burying Test}

Marble burying test is used to assess the Obsessive Compulsive behaviour and is done using polycarbonate rat cages $(26 \mathrm{~cm} \times 48 \mathrm{~cm} \times 20 \mathrm{~cm})$ with bedding material to a depth of $5 \mathrm{~cm}$ and placed standard glass toy marbles (assorted styles and colours, $15 \mathrm{~mm}$ diameter, $5.2 \mathrm{~g}$ in weight) gently on the surface of the bedding in 5 rows of 4 marbles. Rats were placed into a corner of the cage containing marbles and remained for 30 minutes and measured the number of marbles buried in bedding materials (12).

\section{Hole Board Test}

The hole board test is used to assess the fear and anxiety of an animals. The apparatus consists of wooden box $(68 \mathrm{~cm} \mathrm{X} 68 \mathrm{~cm})$ and the walls were raised $(28 \mathrm{~cm})$ from the ground and in centre of the box contained four holes (4 $\mathrm{cm}$ in DM). In habituation, animals kept in centre area of the box for $10 \mathrm{~min}$. After the trial, animals were kept for 10 minutes and measure the number of head dipping in to the four holes. (13).

\section{Histology}

The rats were sacrificed by cervical dislocation. After sacrifice, the brain was rapidly removed, and the hippocampus were dissected on an ice-cold plate and the weight of the discrete region of the brain was measured. Another set of rats were subjected to transcardiac perfusion and fixed in $10 \%$ neutral formalin for $48 \mathrm{~h}$ solution followed by dehydration in ascending grades of alcohol, cleared and then embedded in paraffin wax. Paraffin block $5 \mu \mathrm{m}$ thick coronal sections were obtained using a rotary microtome, and mounted on slides, followed by Hematoxylon and Eosin and Congo red staining(14). Photomicrographs were obtained using a Nikon Camera (Japan).

\section{DNA Fragmentation Assay}

The isolation of DNA from animal tissue was done according to the method of Iwasa et al., (1996). The hippocampal tissue was homogenized in TE buffer and suspension was treated with lysis buffer and kept in a water bath for 12 hours at $37^{\circ} \mathrm{C}$. DNA was extracted twice with equal volume of a Phenol:Choloroform: Isoamylalcohol. To the aqueous phase $1 / 10$ volume of $3 \mathrm{M}$ sodium acetate was added and DNA was receipted with 0.7 volume of chilled isopropanol. Following a $70 \%$ ethanol, the precipitated DNA was resuspended in $50 \mu 1$ of TE buffer. DNA samples were electrophoresed in $0.8 \%$ agarose gel containing ethidium bromide and the gel was examined under UV light and documented(15).

\section{Statistical Analysis}

Data are expressed as Mean \pm Standard deviation (SD). Statistical significance between different groups was determined by one way-Analysis of Variance (ANOVA). The results are shown by using Graph Pad Prism software (Version 9). Tukey's multiple comparison test was done. The significance level was fixed at $\mathrm{p}<0.05$.

\section{Results and Discussion}

One gram of ethanolic extract contains 3.417 $\mu \mathrm{g}$ of phenolic compounds, $428 \pm 38 \mu \mathrm{g}$ of Flavonoids, and $208 \pm 16 \mu \mathrm{g}$ of Carotenoid. Husain et al., 2015 also reported that, phytochemical screening of the ethanolic extract of Glycyrrhiza glabra root revealed the presence of alkaloids, glycosides, carbohydrates, starches, phenolic compounds, flavonoids, proteins, pectin, mucilage, saponins, lipids, tannins, sterols and steroid(16).Chronic stress shown reduction of the whole brain (Figure 1) and Hippocampal weight (Figure 2) of the animals when compared to the control animals and Noise + GG shown statistically increased whole brain weight and hippocampal weight when compared to the chronic noise group animals. Whole brain weight and hippocampal weight was reduced in chronic noise induced animals. Brain cells are at particular risk of being damaged by free radicals because the brain has a 
high oxygen turnover and neuronal membranes in central nervous system (CNS) are rich in polyunsaturated fatty acids, which are the potential targets for lipid peroxidation. An imbalance of the equilibrium between free radical generation and various antioxidant defense systems may lead to the accumulation of free radicals causing neuronal cell death(17). GG treated groups showed increased whole brain weight and hippocampal weight in noise stress animals. This may be due to anti-inflammatory activity that might have improved the hippocampal CA3 neuronal dendritic arborization by GG root treatment $(150 \mathrm{mg} / \mathrm{kg} \mathrm{BW})$ and this was further confirmed by(18).

The numbers of marbles buried (Figure 3 ) in the case of animals exposed to chronic noise were significantly greater when compared to the control animals and significantly reduced in noise + GG when compared to the stress group. Number of head dipping (Figure 4) reduced in chronic stress animals when compared to the control animals and increased in noise + GG. Reduction of hippocampal volume causes behavioral changes such as obsessive compulsive and increased fear and anxiety in animals. The hippocampus has lately been determined to play a vital role in the pathology of adult obsessive-compulsive disorder (OCD)(19)and also Atamaca et al reported that hippocampus, basolateral amygdala and anterior cingulate interrelated together which may have role in the pathology of OCD and reduction of hippocampal volume to $12 \%$ in AD patients(20). Ambawade et al., 2001 also reported that hydroalcoholic extract of GG exhibited potent anxiolytic activity(21). The bioactive compound of GG is ISL, a flavonoid chalcone, easily passes through the blood brain barrier which suggesting that liquiritigenin attenuates anxiety-like behavior and discovered to have neuroprotective effects(22).

In histological studies, sections obtained from brain of chronic noise stress animals exhibited shrinking and enlargement of lateral ventricle when compared to the control brain (Figure 5). Congo red staining methods are helpful for histological identification of pathological deposits. In the control specimens, the brain neurons were stained by hematoxylin and absence of the congo red and in case of chronic stress exposed specimens, they were reddish -pink colored cell body, neurofibrillary tangles and neuropil threads were more readily apparent (Figure 6). Sharifabad et al 2008 also suggested that chronic noise exposure significantly reduced the hippocampal volume in all the layers of dentate gyrus and CA regions(23). The hippocampal cells express the glucocorticoid receptors (GRs), they are the principal target sites for glucocorticoids which are the adrenocortical hormones secreted during stress(24). Activation of $\mathrm{GC}$ receptors leads to the overproduction of ROS(25), increases oxidative damage to the protein due to the inhibiting activities of mitochondrial complex I and antioxidant enzyme SOD(26). Conrad reported that chronic stress or prolonged exposure to glucocorticoids can compromise the hippocampus by producing dendritic retraction, a reversible form of plasticity that includes dendritic restructuring without irreversible cell death(27).In congo red staining, Presence of $A ß$ might be because of chronic noise exposure that lead to $A \beta$ accumulation and would have induced a pro-inflammatory response that includes TNF- $\alpha$ and RAGE overexpression in the hippocampus(28). BACE1 is the rate-limiting enzyme for $A \beta$ production. $G G$ and its bioactive components contains specific BACE1 inhibitor and so has the ability to cross the blood-brain barrier(29), (30) Amyloid deposits were present throughout the hippocampus in stress animals.

In DNA fragmentation chronic noise shown increased DNA fragments when compared to noise + GG and GG alone groups (Fig 7). Noise exposure has been reported to increase $\operatorname{ROS}(31)$. The persistence of ROS mediated DNA alterations might lead to serious and long lasting consequences, as suggested by the association between the persistence of ROS mediated DNA alterations and mutagenic events(32). DNA damage might be interpreted as the output of the clastogenic effect of oxiradicals(33). The involvement of ROS might play a causal role in the induction and persistence of genetic damage related to loud noise exposure also in extra-auditory organs(34). Treatment with GG did not alter the DNA integrity. Study demonstrated that pretreatment with vitamin $\mathrm{C}$ and vitamin $\mathrm{E}$ prevented the alcohol induced DNA strand break. From this view, GG contains enormous amount of antioxidants and enhanced the vitamin $\mathrm{C}$ and vitamin E level which may be prevent the DNA degradation in noise stress treated with $\mathrm{GG}(35)$.

\section{Conclusion}

Noise beyond the bearable limit would make us feel upset and even get frustrated. When sound exceeds $90 \mathrm{~dB}$, it leads to deterioration of the health of an individual. The term "Noise stress" is a highly prevalent concept in the challenging and highly-demanding fashionable society currently. In conclusion, our results confirmed that chronic noise stress led to a negative impact on the hippocampus and its functions in albino rats and Glycyrrhiza glabra has a potent neuroprotective effect on stress induced hippocampal damage.

\section{Funding}

This research did not receive any specific grant from funding agencies in the public, commercial, or not-for-profit sectors.

\section{Author Contributions}

Archana Arjunan and Dr. Ravindran Rajan conceived and designed the study. Archana Arjunan performed the experiments and analyzed the data. Dr. Ravindran Rajan and Dhiraj Kumar Sah drafted the manuscript. All authors read and approved the final manuscript.

\section{Acknowledgement}

The authors are grateful to the Department of Physiology and Multi-Disciplinary Research Unit (MRU), University of Madras, Dr. A.L.M Post Graduate 
Institute of Basic Medical Sciences, Taramani, and Chennai - 600113 for providing the infrastructure to conduct the research.

\section{Conflict of Interest}

The authors declare that they no conflicts of interest concerning this research article.

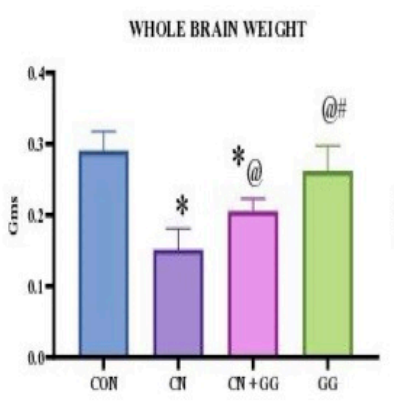

Fig. 1

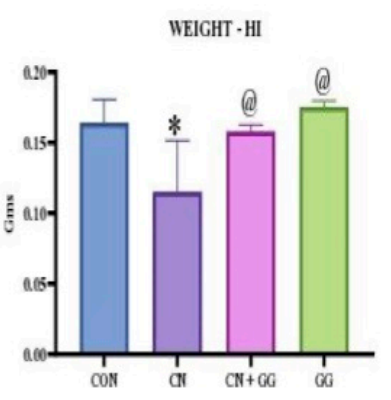

Fig. 2
HOLE BOARD TEST

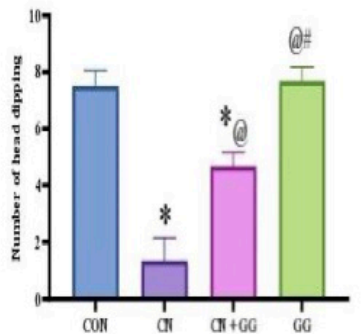

Fig. 3

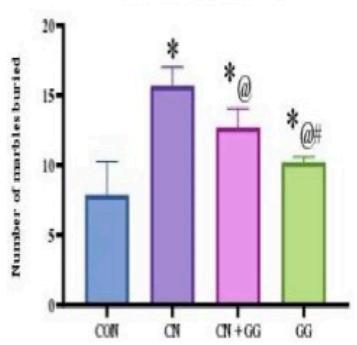

Fig. 4

Lateral Ventricles (Fig 5(A-D) (2X)

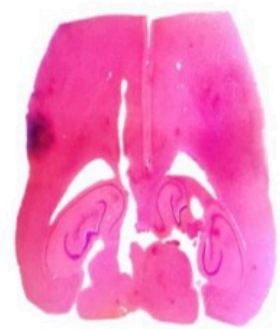

Fig 5A Control

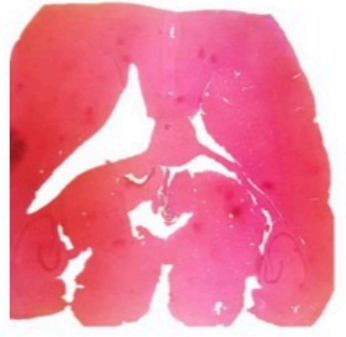

Fig 5B Chronic Noise

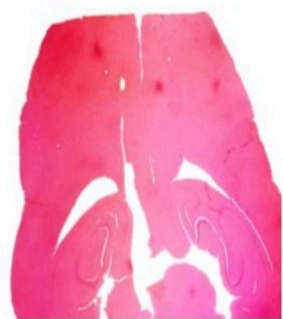

Fig 5C Chronic Noise + GG

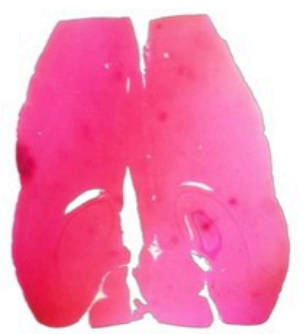

Fig 5D GG

(10 X)
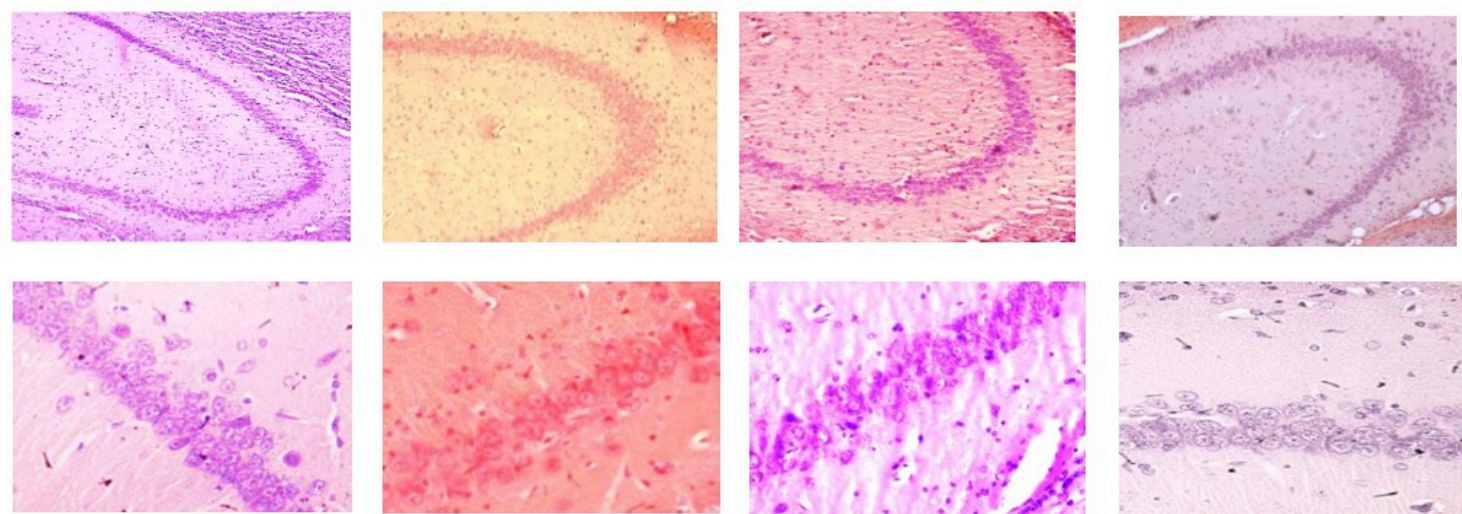

Fig 6 (A) Control

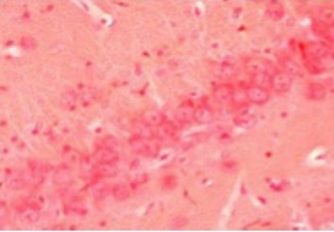

Fig 6 (B) Chronic Noise

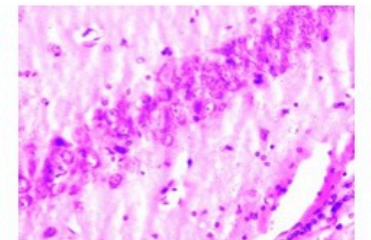

Fig 6(C)Chronic Noise + GG

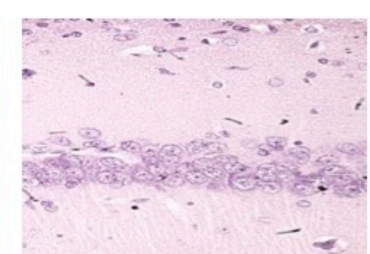

Fig 6 (D) GG

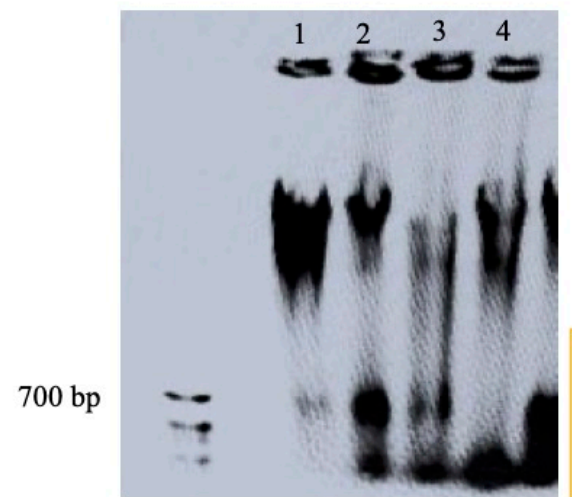

Fig 7 DNA Fragmentation

Lane 1: Control

Lane 2: Chronic Noise + GG

Lane 3: Chronic Noise

Lane 4: GG

All data were reported as Mean $\pm \mathrm{SD}$. One way ANOVA was used for statistical analysis $\mathrm{p}<0.05$ considered as significant.

- - when compared to the control, @ - when compared to the chronic Noise, \# - when compared to the Chronic Noise + GG. 


\section{References}

1. Asch A. and Marmor R., "B ioethics B riefing B ook," In Vitro, 2008.

2. Salter C. M. et al., "Community noise, urbanization, and global health: Problems and solutions," in Innovating for Healthy Urbanization, 2015.

3. Krugers H J, Lucassen P. J., Karst H., and Joëls M., "Chronic stress effects on hippocampal structure and synaptic function: Relevance for depression and normalization by anti-glucocorticoid treatment," Front. Synaptic Neurosci., 2010, doi: 10.3389/ fnsyn.2010.00024.

4. Wible C.G., "Hippocampal physiology, structure and function and the neuroscience of schizophrenia: A unified account of declarative memory deficits, working memory deficits and schizophrenic symptoms," Behavioral Sciences. 2013, doi: 10.3390/bs3020298.

5. McEwen B. S. and Morrison J. H., "The Brain on Stress: Vulnerability and Plasticity of the Prefrontal Cortex over the Life Course," Neuron. 2013, doi: 10.1016/j.neuron.2013.06.028.

6. Ahmed-Farid O. A., Haredy S. A., Niazy R. M., Linhardt R. J., and Warda M., "Dose-dependent neuroprotective effect of oriental phyto-derived glycyrrhizin on experimental neuroterminal norepinephrine depletion in a rat brain model," Chem. Biol. Interact., 2019, doi: 10.1016/ j.cbi.2019.05.045.

7. Dhingra D, Parle M, and Kulkarni S. K., "Comparative Brain Cholinesterase-Inhibiting Activity of Glycyrrhiza glabra, Myristica fragrans , Ascorbic Acid, and Metrifonate in Mice ," J. Med. Food, 2006, doi: 10.1089/jmf.2006.9.281.

8. Samson J, Devi R.S, Ravindran R, and Senthilvelan $\mathrm{M}$, "Effect of noise stress on free radical scavenging enzymes in brain," Environ. Toxicol. Pharmacol., 2005, doi: 10.1016/j.etap.2004.12.059.

9. Harborne J B, "The Biochemical Systematics of Flavonoids," in The Flavonoids, Springer US, 1975, pp. 1056-1095.

10. Price M. L, Hagerman A.E, and Butler L. G, "Tannin Content of Cowpeas, Chickpeas, Pigeon Peas, and Mung Beans," 1980. (Online). Available: https://pubs.acs.org/sharingguidelines.

11. Narayanasamy P and Palaniswami A, "Studies on yellow mosaic disease of soybean. I. Effect of virus infection on plant pigments," Experientia, vol. 29, no. 9, pp. 1166-1167, Sep. 1973, doi: 10.1007/ BF01946787.

12. Angoa-Pérez M, Kane M.J, Briggs D.I, Francescutti D.M, and Kuhn D.M, "Marble Burying and Nestlet Shredding as Tests of Repetitive, Compulsive-like Behaviors in Mice," J. Vis. Exp., 2013, doi: 10.3791/50978.

13. Brown G.R and Nemes C, "The exploratory behaviour of rats in the hole-board apparatus: Is head-dipping a valid measure of neophilia?," Behav. Processes, 2008 , doi: 10.1016/ j.beproc.2008.02.019.
14. Spencer LT and Bancroft JD, "Tissue Processing," in Bancroft's theory and practice of histological techniques, 2013.

15. Iwasa $M$, Maeno $Y$, Inoue $H$, Koyama $H$, and Matoba R, "Induction of apoptotic cell death in vat thymus and spleen after a bolus injection of methamphetamine," Int. J. Legal Med., vol. 109, no. 1, pp. 23-28, 1996, doi: 10.1007/BF01369597.

16. Husain A and Ahmad A, "Quantitative Analysis of Total Phenolic, Flavonoid Contents and HPTLC Fingerprinting for Standardization of Glycyrrhiza glabra Linn. Roots," Herb. Med. Open Access, 2015, doi: 10.21767/2472-0151.10001.

17. Behl C, Davis J B, Lesley R, and Schubert D, "Hydrogen peroxide mediates amyloid $\beta$ protein toxicity," Cell, vol. 77, no. 6, pp. 817-827, Jun. 1994, doi: 10.1016/0092-8674(94)90131-7.

18. Chakravarthi K and Avadhani R, "Beneficial effect of aqueous root extract of Glycyrrhiza glabra on learning and memory using different behavioral models: An experimental study," J. Nat. Sci. Biol. Med., 2013, doi: 10.4103/0976-9668.117025.

19. Reess T J et al., "Association between hippocampus volume and symptom profiles in obsessivecompulsive disorder," NeuroImage Clin., 2018, doi: 10.1016/j.nicl.2017.11.006.

20. Atmaca $\mathrm{M}$ et al., "Hippocampus and amygdalar volumes in patients with refractory obsessivecompulsive disorder," Prog. NeuroPsychopharmacology Biol. Psychiatry, 2008, doi: 10.1016/j.pnpbp.2008.04.002.

21. Ambawade S D, Kasture V S, and Kasture S B, "ANTICONVULSANT ACTIVITY OF ROOTS AND RHIZOMES OF GLYCYRRHIZA," Indian J. Phamacology, 2002.

22. Wang D, Liang J, Zhang J, Wang Y, and Chai X, "Natural Chalcones in Chinese Materia Medica: Licorice," Evidence-based Complementary and Alternative Medicine, vol. 2020. Hindawi Limited, 2020, doi: $10.1155 / 2020 / 3821248$.

23. Hosseini-Sharifabad M and Sabahi A, "Exposure to chronic noise reduces the volume of hippocampal subregions in rats," Iran. J. Basic Med. Sci., 2008, doi: 10.22038/IJBMS.2008.5192.

24. Behl C, Lezoualc'h F, Trapp T, Widmann M, Skutella T, and Holsboer F, "Glucocorticoids enhance oxidative stress-induced cell death in hippocampal neurons in vitro," Endocrinology, 1997, doi: 10.1210/endo.138.1.4835.

25. You J M, Yun S J, Nam K N, Kang C, Won R, and Lee E H , "Mechanism of glucocorticoid-induced oxidative stress in rat hippocampal slice cultures," Can. J. Physiol. Pharmacol., 2009, doi: 10.1139/ Y09-027.

26. Tang V M, Young A H, Tan H, Beasley C, and Wang J F, "Glucocorticoids increase protein carbonylation and mitochondrial dysfunction," Horm. Metab. Res., 2013, doi: 10.1055/ s-0033-1345119.

27. Conrad C D, "Chronic stress-induced hippocampal vulnerability: The glucocorticoid vulnerability 
hypothesis," Reviews in the Neurosciences. 2008 doi: 10.1515/REVNEURO.2008.19.6.395.

28. Cui B et al., "Chronic Noise Exposure Acts Cumulatively to Exacerbate Alzheimer's DiseaseLike Amyloid- $\beta$ Pathology and Neuroinflammation in the Rat Hippocampus," Sci. Rep., 2015, doi: $10.1038 /$ srep 12943.

29. Zhu Z et al., '2,2',4'-Trihydroxychalcone from Glycyrrhiza glabra as a new specific BACE1 inhibitor efficiently ameliorates memory impairment in mice," J. Neurochem., 2010, doi: 10.1111/j.1471-4159.2010.06751.x.

30. Yang R, Yuan B C, Ma Y S, Zhou S, and Liu Y, "The anti-inflammatory activity of licorice, a widely used chinese herb," Pharmaceutical $\begin{array}{rr}B \text { i o } l o g y \text {. } & 2017 \text {, } \\ 10.1080 / 13880209.2016 .1225775 .\end{array}$

31. Manikandan S and Devi R S, "Antioxidant property of $\alpha$-asarone against noise-stress-induced changes in different regions of rat brain," Pharmacol. Res., 2005, doi: 10.1016/j.phrs.2005.07.007.
32. Emerit I, "Reactive oxygen species, chromosome mutation, and cancer: possible role of clastogenic factors in carcinogenesis," Free Radical Biology and Medicine, vol. 16, no. 1. Pergamon, pp. 99$109, \quad$ J a n. $01, \quad 1994, \quad$ d o i : 10.1016/0891-5849(94)90246-1.

33. Frenzilli G et al., "DNA damage in eelpout (Zoarces viviparus) from Göteborg harbour," Mutat. Res. - Fundam. Mol. Mech. Mutagen., vol. 552, no. 1-2, pp. 187-195, Aug. 2004, doi: 10.1016/ j.mrfmmm.2004.06.018.

34. Van Campen L W, Murphy W J, and Toraason M A, "Oxidative DNA damage associated with intense noise exposure in a rat," J. Acoust. Soc. Am., vol. 109, no. 5, pp. 2373-2373, May 2001, doi: 10.1121/1.4744353.

35. Navasumrit P, "Ethanol-induced free radicals and hepatic DNA strand breaks are prevented in vivo by antioxidants: effects of acute and chronic ethanol exposure," Carcinogenesis, vol. 21, no. 1, pp. 9399, Jan. 2000, doi: 10.1093/carcin/21.1.93. 